\title{
The role of natural convection and density variations in the solidification process of water in an annular enclosure
}

\author{
E. M. Alawadhi \& R. I. Bourisli \\ Mechanical Engineering Department, Kuwait University, Kuwait
}

\begin{abstract}
Unsteady natural convection flow during the solidification process of water in the annulus is numerically modeled using the finite element method. The annulus inner surface temperature is kept at a temperature lower than the solidification temperature. Ice is formed at the inner surface while natural convection flow is induced in the liquid portion. The goal of this study is to evaluate the effect of natural convection and variations in the density of water on the solidification process. The water density peak near the solidification temperature creates a unique flow structure. High resolution capturing of the solid/liquid moving boundary and the details of the flow structure and temperature contours are presented.

Keywords: natural convection, solidification, density variation, annulus enclosure.
\end{abstract}

\section{Introduction}

Phase change heat transfer has been extensively studied experimentally and theoretically in literature during the past two decades. Phase change has a wide range of utility in scientific and engineering applications. These applications include, for example, solar energy storage units, industrial refrigeration, crystal growth, welding and casting. During melting process, the effective specific heat of a substance is increased by more than 100 times, storing a large amount of energy. On the other hand, during solidification process, the specific heat is decreased to release a large amount of energy. Phase-change materials (PCM's) are substances that are used to store/release thermal energy by utilizing this effective specific heat 
variation. Small but influential variations in density often accompany this process and can have detrimental impact on the solidification process.

Common PCM's include waxes, many types of salts, as well as water. Wax PCM's are extensively employed in electronic devices as a method of thermal management [1], and in thermal control units [2]. In electronic cooling, PCM's are used to absorb heat from high thermal dissipating components during operating times, and then, release the stored energy during OFF times [3]. In thermal storage systems, especially in solar storage units, water is commonly used to store energy during high-energy demand periods [4]. Understanding phase change heat transfer mechanism in an enclosure is essential to predict the thermal performance of a PCM thermal unit.

Modeling the melting and solidification processes within a fixed enclosure has been of special interest to engineers. Modeling such processes can be intricate because of the need to deal with conduction in the solid portion, and conduction plus convection in the liquid portion. The phenomena are strongly coupled and the modeling results are extremely sensitive to the set up as well as the physics of the problem. Giangi et al. [5] performed numerical and experimental studies on natural convection flow during solidification of water in a rectangular enclosure with constant temperature boundary conditions. PCM melting in a rectangular enclosure by heating the bottom side was also studied by Gong and Mujumdar [6]. The resulting complex flow patterns were consistent with experimental results. Phase change heat transfer for a pure metal in a rectangular enclosure was studies by Mbaye and Bilgen [7]. Their results indicated that the Nusselt number passed through the maximum when the enclosure aspect ratio is unity. A rectangular composite cell filled with PCM and water was studied by Ho and Chu [8], and the effect of an ON/OFF pulsing of boundary heat load was analyzed. El Omari et al. [9] also studied the impact of the shape of the enclosure on the PCM solidification process, and found that non-slender shapes provide little control over the process.

More importantly, and more relevant to the subject of this work, Pal and Joshi [10] investigated the effect of natural convection on PCM melting rate in a honeycomb enclosure. When the PCM in the system is relatively small, or the PCM is placed in a metal matrix, natural convection flow in the liquid portion is often neglected, the rationale being that conduction dominates in the overall domain. While this approximation is widely used in phase change simulations [11-13], it could produce significant error. This also related to the variation in the density of the material as it changes temperature and phase. Kuznetsov and Sheremet [14] numerically studied the influence of Rayleigh number on fluid motion and heat (and mass) transfer in enclosures. They related these quantities specifically to the dimensional time and outlined regions of variations in the general behavior of flow as a result of phase change.

In this research, solidification process of pure water in an annulus enclosure is investigated. The annulus enclosure represents a practical geometry for thermal storage pipes [15]. Water is commonly used in thermal storage units, because of its availability, non-toxicity, non-flammability, and chemical stability. In addition, its 
latent heat of fusion is relatively high compared to other PCM's. Initially, the water temperature in the annulus is higher than solidification temperature. At a certain instant, the boundary temperature at the inner surface is decreased to temperature lower than the solidification temperature. Ice is formed at the inner surface, and density gradients in the liquid portion induce natural convection flow. The main objective of this paper is to evaluate the significance of the natural convection effect on the solidification time.

\section{Problem formulation}

A schematic diagram of the model that was used for numerical simulations is shown in figure 1. As shown, it consists of a two-dimensional annulus enclosure of outer radius $r_{o}$ and inner radius $r_{i}$, with $r_{o}=2 r_{i}$. The enclosure is initially at a temperature $T_{i n i}=38^{\circ} \mathrm{C}$, well-higher than water's solidification temperature, $T_{s}=0^{\circ} \mathrm{C}$. At a certain instant $t=0$, the annulus inner wall temperature is dropped to $T_{w}=-2^{\circ} \mathrm{C}$, which is lower than the water solidification temperature. The peakdensity temperature of water is denoted by $T_{p}=4^{\circ} \mathrm{C}$.

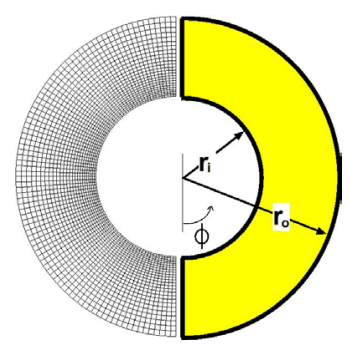

Figure 1: Problem schematics and mesh of annular enclosure.

\subsection{Governing equations}

Phase change simulations were done using apparent capacity paradigm. The apparent capacity is the sum of the sensible and latent heats, as follows

$$
\bar{C}_{p}=C_{p}+\frac{\partial L}{\partial T}
$$

where $C_{p}$ is the specific heat, and $\partial L / \partial T$ is the released latent heat. The apparent capacity can be approximated if the phase change transient temperature is finite, as follows:

$$
\bar{C}_{p}=C_{p}+\frac{\Delta L}{\Delta T}
$$

The value of $\partial L / \partial T$ is equal to zero in the liquid and solid phases. 
The governing equations are nondimensionalized using the following parameters: Rayleigh number $R a$, Prandtl number $\mathrm{Pr}$, Stefan number $S t$, and Fourier number $F o$; they are defined, respectively, as,

$$
R a=\frac{g \beta \Delta T r_{o}^{3}}{\nu \alpha}, \quad \operatorname{Pr}=\frac{\nu}{\alpha}, \quad \text { Ste }=\frac{C_{p} \Delta T}{L}, \quad \text { Fo }=\frac{\alpha t}{r_{o}^{2}}
$$

Based on these dimensionless groups, the non-dimensional forms of the continuity, $x$-momentum, $y$-momentum, and energy equations are obtained as follows,

$$
\begin{gathered}
\frac{\partial u^{\star}}{\partial x^{\star}}=0 \\
\frac{\partial u^{\star}}{\partial F_{O}}+u^{\star} \frac{\partial u^{\star}}{\partial x^{\star}}+v^{\star} \frac{\partial u^{\star}}{\partial y^{\star}}=-\frac{\partial p^{\star}}{\partial x^{\star}}+\operatorname{Pr}(\theta)\left[\frac{\partial^{2} u^{\star}}{\partial x^{\star 2}}+\frac{\partial^{2} u^{\star}}{\partial y^{\star 2}}\right] \\
\frac{\partial v^{\star}}{\partial F_{O}}+u^{\star} \frac{\partial v^{\star}}{\partial x^{\star}}+v^{\star} \frac{\partial v^{\star}}{\partial y^{\star}}=-\frac{\partial p^{\star}}{\partial v^{\star}}+\operatorname{Pr}(\theta)\left[\frac{\partial^{2} v^{\star}}{\partial x^{\star 2}}+\frac{\partial^{2} v^{\star}}{\partial y^{\star 2}}\right]+\operatorname{RaPr} \theta \\
\Phi(\text { Ste, } \theta) \frac{\partial \theta}{\partial F_{O}}+u^{\star} \frac{\partial \theta}{\partial x^{\star}}+v^{\star} \frac{\partial \theta}{\partial y^{\star}}=\frac{\partial^{2} \theta}{\partial x^{\star 2}}+\frac{\partial^{2} \theta}{\partial y^{\star 2}}
\end{gathered}
$$

where

$$
\Phi(\text { Ste }, \theta)= \begin{cases}1, & \theta \leq \theta_{s} \\ 1 / \text { Ste }, & \theta_{s}<\theta<\theta_{s}+\Delta \theta \\ 1, & \theta \geq \theta_{s}+\Delta \theta\end{cases}
$$

Star-superscripted symbols signify dimensionless quantities where the following were used in the nondimensionalization,

$$
\begin{gathered}
x^{\star}=\frac{x}{r_{o}}, \quad y^{\star}=\frac{y}{r_{o}}, \quad u^{\star}=\frac{u}{\alpha / r_{o}}, \quad v^{\star}=\frac{v}{\alpha / r_{o}}, \\
p^{\star}=\frac{p}{\rho \alpha^{2} / r_{o}^{2}}, \quad \theta=\frac{T-T_{w}}{\left|T_{s}-T_{w}\right|}
\end{gathered}
$$

Finally, initial and boundary conditions are written in dimensional forms, using the dimensionless time $\tau$, as,

$$
\begin{array}{rlll}
\tau=0: & u^{\star}=v^{\star}=0 & \text { and } & \theta=\theta_{0}=20 \\
\tau>0, r=r_{i}: & u^{\star}=v^{\star}=0 & \text { and } & \theta=\theta_{w}=0 \\
\tau>0, r=r_{o}: & u^{\star}=v^{\star}=0 & \text { and } & \frac{\partial \theta}{\partial n}=0
\end{array}
$$




\subsection{Finite element model}

The finite element model is used to solve simultaneously the set of coupled governing equations for this viscous flow. A four-node quadrilateral element is used to discretize the computational domain, as shown in figure 1 . The finite element model consists of 2,250 elements and 2,366 nodes. The computational domain is reduced by half after taking advantage of symmetry in the problem.

Kuehn and Goldstein [16] conducted an experimental study of natural convection without phase change in a cylindrical annulus. The present code was validated by simulating natural convection in an annulus for the conditions presented by Kuehn and Goldstein. The inner and outer radii of the annulus are $0.0463 \mathrm{~m}$ and $0.0178 \mathrm{~m}$, respectively. The working fluid is air. The inner and outer

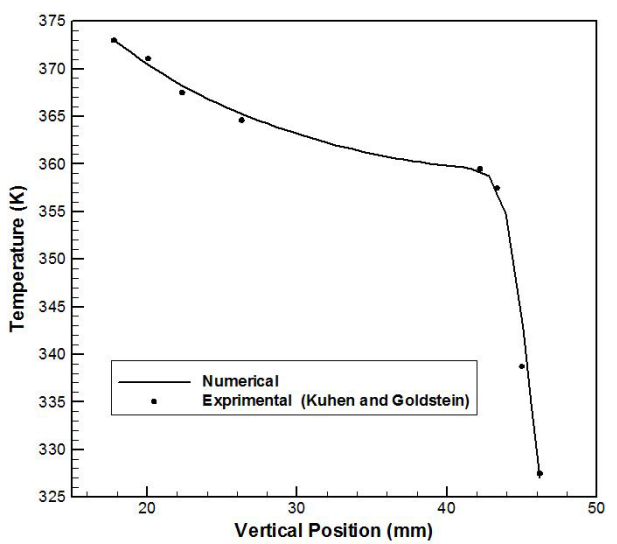

(a)

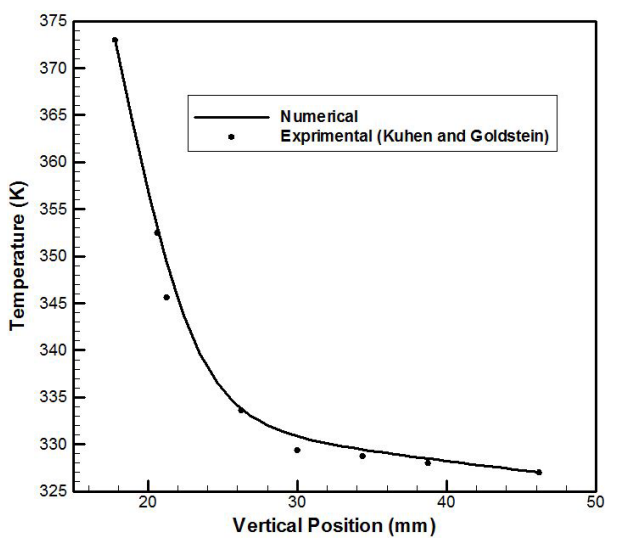

(b)

Figure 2: Comparison of temperature along the top symmetry line for natural convection flow in an annulus. 
cylinder walls are set to temperatures of 373 and $327 \mathrm{~K}$, respectively. The Rayleigh number is $4.95 \times 10^{4}$. The temperature profiles long the top symmetry line of the numerical results and the experimental data are shown in figure 2 . As shown, the agreement between the numerical and experimental results is excellent.

\section{Results and discussion}

Water is selected as the PCM for its common use in thermal storage pipes. Its thermo-physical properties are listed in table 1. Water exhibits a negative volume expansion coefficient at a temperature below $4^{\circ} \mathrm{C}$ and a positive volume expansion above $4^{\circ} \mathrm{C}$. This phenomenon creates a unique flow structure during water solidification. The effect of free convection flow in the liquid portion during the solidification process is examined in the following sections.

Table 1: Thermo-physical properties of water [5].

\begin{tabular}{|l|l|}
\hline Specific heat $\left(C_{p}\right)$ & $4.202 \mathrm{~kJ} / \mathrm{kg}-\mathrm{K}$ \\
\hline Thermal conductivity $(k)$ & $0.56 \mathrm{~W} / \mathrm{m}-\mathrm{K}$ (liquid) \\
& $2.26 \mathrm{~W} / \mathrm{m}-\mathrm{K}$ (solid) \\
\hline Dynamic viscosity & $1.53 \times 10^{-3}$ Pa-s \\
\hline Latent heat $(\mathrm{L})$ & $335 \mathrm{~kJ} / \mathrm{kg}-\mathrm{K}$ \\
\hline Density $(\rho)$ & $999.84+0.067326 T-0.008944 T^{2}$ \\
& $+8.87 \times 10^{-5} T^{3}-6.62 \times 10^{-7} T^{4}$ \\
\hline
\end{tabular}

\subsection{Flow field and temperature contours}

Figures 3 show the velocity streamlines of the flow field alongside the resulting temperature contours at four time steps: $\tau=0.05,0.1,0.15$ and 0.25 , for $R a=$ $10^{7}$. Each curve in the plot on the right-hand-side of these figures represents a streamline, while each curve on the left-hand-side represents an isotherm. Initially, water is in liquid phase $\left(\theta_{0}=20\right)$. At $\tau>0$, the inner wall temperature is dropped to a temperature lower than the water solidification temperature $\left(\theta_{w}=0\right)$.

Figure 3(a) shows how one recirculating cell is formed and the temperature of water is higher than its peak-density temperature of $\theta_{p}=3$. Cold water adjacent to the inner cylinder is continually replaced by warm water coming from the outer cylinder. The water stream is cooled as it flows naturally close to the inner cylinder to leave the inner to outer cylinder. Therefore, the temperature differences between the inner cylinder and the water is maximum at the upper region, and minimum at the lower region. The variations of the temperature difference explain the observed wide temperature gradient at the upper region of the annulus. Up to this time 


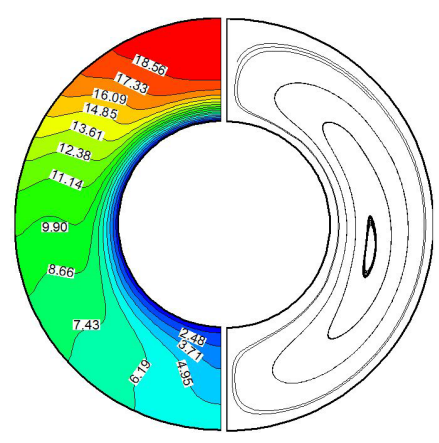

(a)

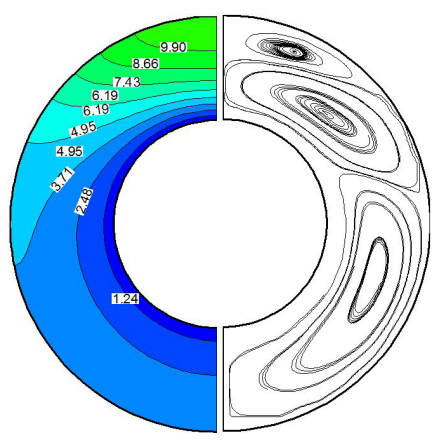

(c)

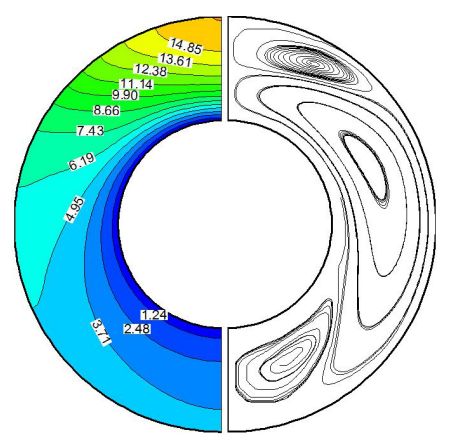

(b)

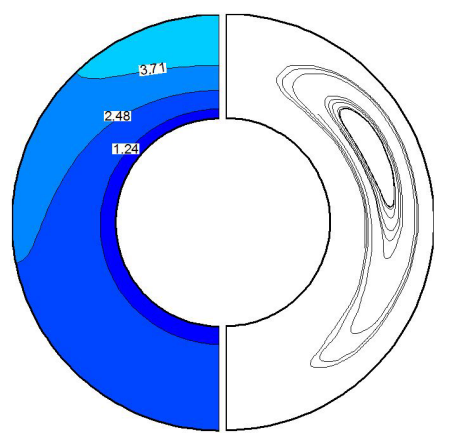

(d)

Figure 3: Flow field streamlines and temperature contour of the solidification process for $R a=10^{7}$ at (a) $\tau=0.05$, (b) $\tau=0.1$, (c) $\tau=0.15$, (d) $\tau=0.25$.

( $\tau=0.05$ ), one recirculating cell exists in the annulus. As time progresses, two additional recirculating cells are formed, as shown in figure 3(b), one at the top and another at the bottom of the original recirculating cell. Furthermore, while the original cell recirculates in the counter clockwise direction, the two newly formed cells have clockwise directions. Water temperature decreases as it flows close to the inner cylinder, and reaches the peak-density temperature. When water temperature becomes less than $\left(\theta_{p}\right)$, water density decreases to create a clockwise flow cell, and therefore, the direction of flow is reversed from counterclockwise to clockwise, creating a small cell below the original cell. The direction of original cell is still counterclockwise, because the temperature of the cell is higher than the peak-density temperature. These results are consistent with those of de Souza and Vielmo [17] who studied ice formation and melting processes in tubes for HVAC applications. 


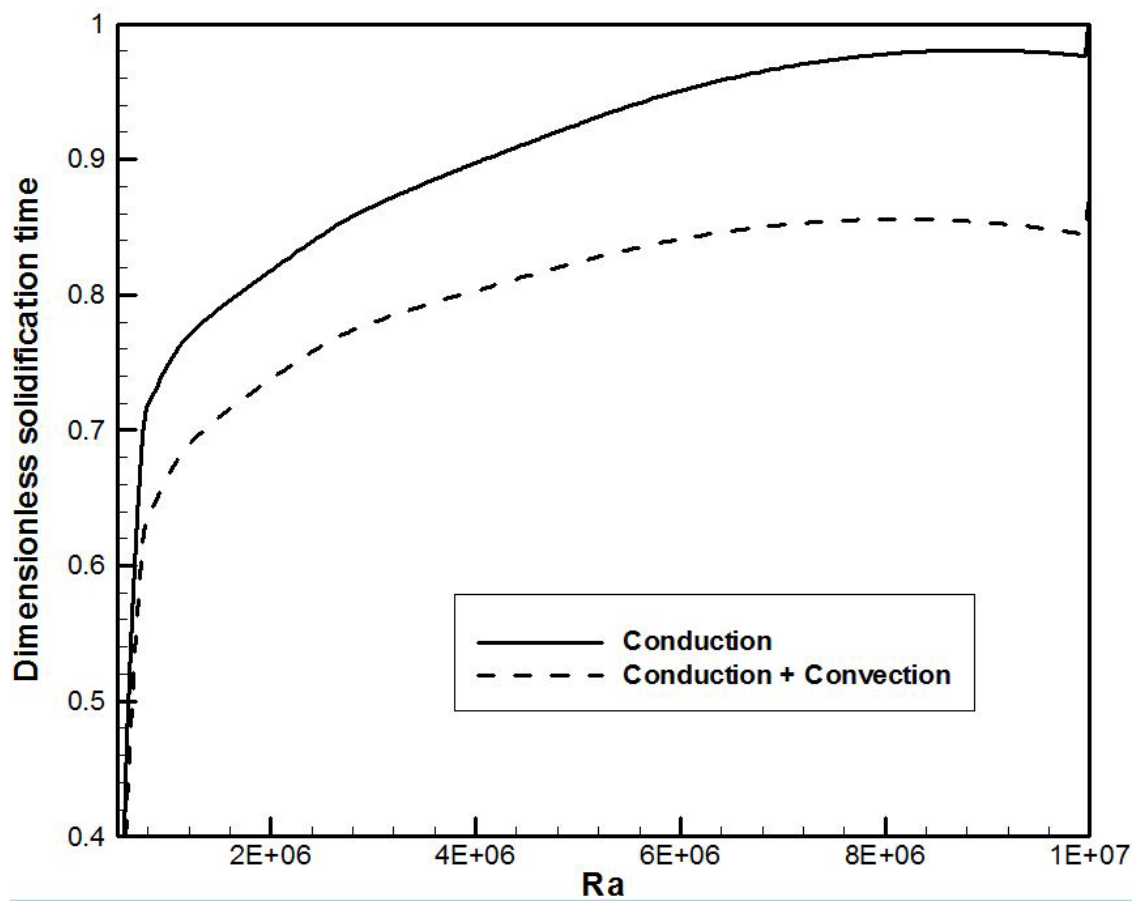

Figure 4: Effect of Rayleigh number on the water solidification time, for conduction and conduction-plus-convection modes of heat transfer, for $R a=10^{7}$.

\subsection{Effect of natural convection flow}

Figure 4 shows the solidification time for the conduction and conduction-plusconvection modes of heat transfer with $R a=10^{6}$ to $10^{7}$. For $10^{6}<R a<$ $1.25 \times 10^{6}$, the solidification times for conduction and conduction-plus-convection are almost the same, showing a negligible effect of the convection as a heat transfer mechanism. Hence, eliminating the natural convection flow during phase change simulation is an acceptable approximation for this range of $R a$. For $R a \geq 1.25 \times 10^{6}$, figure 4 indicates that the effect of natural convection becomes significant. With $R a=10^{7}$, the natural convection decreases the solidification time by $13.3 \%$.

Figures 5 show the average heat flux at the inner cylinder for conduction and conduction-plus-convection for $R a=10^{6}$ and $110^{7}$. For $R a=10^{6}$, figure 5(a) indicates that the heat flux differences between the conduction and conduction plus convection is negligible. The strength of the natural flow is so weak that it does not decrease the thermal resistance in the annulus. On the other hand, when the $R a$ is increase to $10^{7}$, figure $5(\mathrm{~b})$ indicates that the effect of natural convection is only initially insignificant $(\tau<0.15)$ (note the negligible difference between the two 


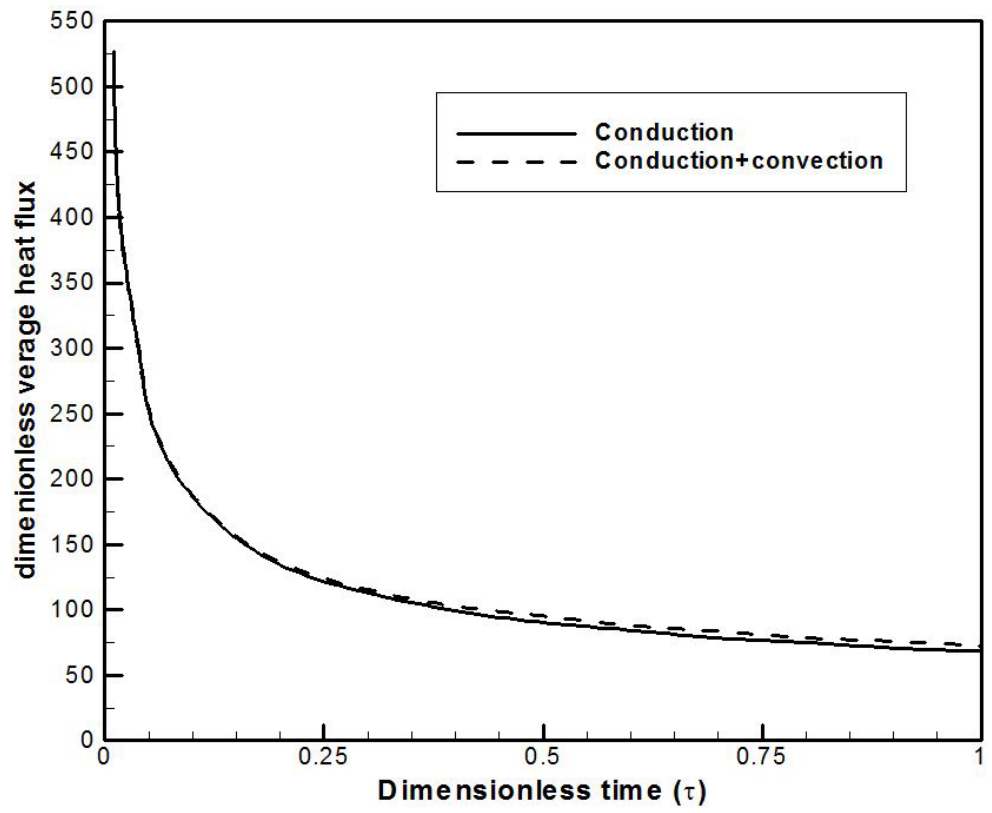

(a)

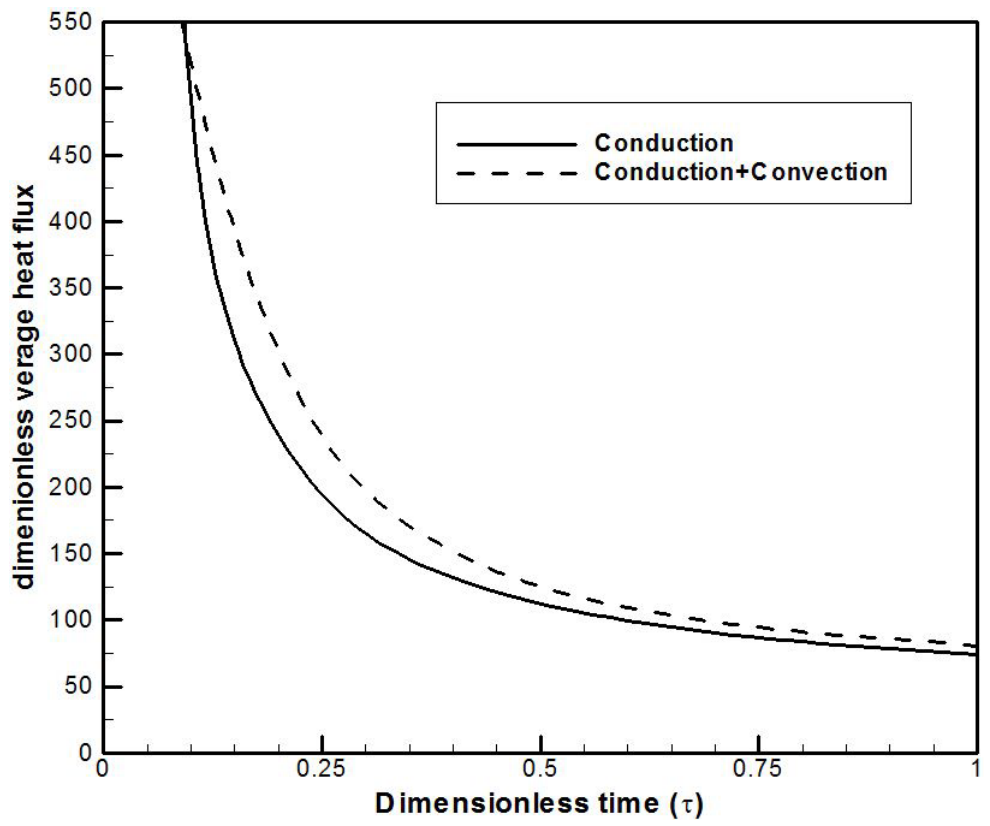

(b)

Figure 5: Variation of average heat flux at the inner cylinder with time, for (a) $R a=10^{6}$ and (b) $R a=10^{7}$ 
cases.) During the period $0.15<\tau<0.6$, however, the natural convection flow increases the heat transfer into the annulus by decreasing the thermal resistance in the annulus. Natural convention flow improves heat flow in the annulus by transporting warm water from the outer boundary to the freezing interface. For $\tau>0.6$, the heat flux of the two cases is close again, indicating that the natural convection flow is slowing down and flow temperature becomes close to freezing temperature.

\section{Conclusions}

The main objective of this paper was to evaluate the effect of natural convection flow during water solidification process. Natural convection is commonly neglected in phase change simulations, even if the Rayleigh number is high. The results indicate that the effect of natural convention flow is significant for a wide range of Rayleigh numbers, and this significance is increased as the Rayleigh number is increased. Specifically, when the Rayleigh number is less than $1.25 \times 10^{6}$, the convection mode of heat transfer is an insignificant factor with regards to the solidification rate. For Rayleigh numbers higher than $1.25 \times 10^{6}$, natural convection flow plays an important role in reducing thermal resistance in the domain, enhancing heat transfer, and increasing the solidification rate. Therefore, neglecting natural convection flow can produce significant errors in numerical simulations.

\section{References}

[1] Ravi Kandasamy, Xiang-Qi Wang, and Arun S. Mujumdar. Transient cooling of electronics using phase change material (PCM)-based heat sinks. Applied Thermal Engineering, 28(8-9):1047-1057, 2008.

[2] D. P. Covin, J. C. Mulligan, and Y. G. Bryant. Enhanced heat transport in environmental systems using microencapsulated phase change materials. In 22nd International Conference on Environmental Systems, 1992.

[3] R. C. Estes. The effect of thermal capacitance and phase change on outside plant electronic enclosure. IEEE Transactions on Components, Hybrids, and Manufacturing Technology, 5:843-849, 1992.

[4] R. Velraj, R. V. Seeniraj, B. Hafner, C. Faber, and K. Schwarzer. Heat transfer enhancement in a latent heat storage system. Solar Energy, 65:171-180, 1999.

[5] Marilena Giangi, Fulvio Stella, and Tomasz A. Kowalewski. Phase change problems with free convection: fixed grid numerical simulation. Computing and visualizeation in science, 2:123-130, 1999.

[6] Zhen-Xiang Gong and Aron Mujumdar. A finite element method for convection-dominated melting and solidification problem. International Journal of Numerical Methods for Heat \& Fluid Flow, 8:393-408, 1998. 
[7] M. Mbaye and E. Bilgen. Phase change process by natural convectiondiffusion in rectangular enclosures. Heat and Mass Transfer, 37:35-42, 2001.

[8] C. Ho and C. Chu. On cooling behavior of vertical planes in a phase change/water composite enclosure under pulsing heat load. International Journal of Heat and Mass Transfer, 34:509-514, 1999.

[9] Kamal El Omari, Tarik Kousksou, and Yves Le Guer. Impact of shape of container on natural convection and melting inside enclosures used for passive cooling of electronic devices. Applied Thermal Engineering, 31(1415):3022-3035, 2011.

[10] D. Pal and Y. Joshi. Thermal management of an avionics model using solidliquid phase change materials. Journal of Thermophysics and Heat Transfer, 256:12-17, 1998.

[11] T. J. Lu. Thermal management of high power electronics with phase change cooling. International Journal of Heat and Mass Transfer, 43:2245-2256, 2000.

[12] Y. Cao and A. Faghri. Performance characteristics of a thermal energy storage module: a transient pcm/forced convection conjugate analysis. International Journal of Heat and Mass Transfer, 34:93-101, 1991.

[13] M. Lacroix. Study of the heat transfer behavior of a latent heat thermal energy storage unit with a finned tube. International Journal of Heat and Mass Transfer, 36:2083-2092, 1993.

[14] Geniy V. Kuznetsov and Mikhail A. Sheremet Applying computer-based simulation to energy auditing: A case study. International Journal of Thermal Sciences, 50(10):1878-1886, 2011.

[15] K. A. R. Ismail and M. M. Goncalves. Thermal performance of a pcm storage unit. Energy Conversion \& Management, 49:115-138, 1999.

[16] T. H. Kuehn and R. J. Goldstein. An experimental study of natural convection heat transfer in concentric and eccentric horizontal cylindrical annuli. Journal of Heat Transfer, Transactions of the ASME, 100:635-640, 1978.

[17] S. I. S. de Souza and H. A. Vielmo. Numerical analysis of water melting and solidification in the interior of tubes. Journal of the Brazilian Society of Mechanical Sciences and Engineering, XXVII(2):119-131, 2005. 